\begin{tabular}{|c|l|}
\hline Title & Dispersion relation in oscillatory reaction-diffusion systems with self-consistent flow in true slime mold \\
\hline Author(s) & Y amada, H.; Nakagaki, T.; Baker, R.E.; Maini, P.K. \\
\hline Citation & $\begin{array}{l}\text { Journal of Mathematical Biology, 54(6), 745-760 } \\
\text { https://doi.org/10.1007/300285-006-0067-1 }\end{array}$ \\
\hline Issue Date & 2007-06 \\
\hline Doc URL & http://hdl.handle.net/2115/28017 \\
\hline Rights & The original publication is available at www.springerlink.com \\
\hline Type & article (author version) \\
\hline File Information & JMB546.pdf \\
\hline
\end{tabular}

Instructions for use 


\title{
Dispersion relation in oscillatory reaction- diffusion systems with self-consistent flow in true slime mold
}

Received: date / Revised: date

\begin{abstract}
In the large amoeboid organism Physarum, biochemical oscillators are spatially distributed throughout the organism and their collective motion exhibits phase waves, which carry physiological signals. The basic nature of this wave behaviour is not well-understood because, to date, an important effect has been neglected, namely, the shuttle streaming of protoplasm which accompanies the biochemical rhythms. Here we study the effects of self-consistent flow on the wave behaviour of oscillatory reaction-diffusion models proposed for the Physarum plasmodium, by means of numerical simulation for the dispersion relation and weakly nonlinear analysis for derivation of the phase equation. We conclude that the flow term is able to increase the speed of phase waves (similar to elongation of wave length). We compare the theoretical consequences with real waves observed in the organism and also point out the physiological roles of these effects on control mechanisms of intracellular communication.
\end{abstract}

Keywords Dispersion relation - Reaction-diffusion-advection equation · Physarum $\cdot$ Phase equation · Amoeboid movement

H. Yamada

Harukidai 5-1-2, Togo, Aichi 470-0161, Japan

E-mail: hyamada@r.phys.nagoya-u.ac.jp

T. Nakagaki

Creative Research Initiative "SOUSEI", Hokkaido University, Sapporo 001-0821, Japan

Tel.: +81-11-706-9211

Fax: +81-11-706-9211

E-mail: nakagaki@es.hokudai.ac.jp

R. Baker and P. K. Maini

Centre for Mathematical Biology, Mathematical Institute, 24-29 St. Giles, Oxford, OX1 3LB, UK

Tel.: +44-1865-283889

Fax: +44-1865-270515

E-mail: ruth.baker@maths.ox.ac.uk,maini@maths.ox.ac.uk 


\section{Introduction}

The amoeboid plasmodium of the true slime mold Physarum polycephalum shows tactic movement which develops due to an underlying mechanism based on wave phenomena arising in an oscillatory field $[13,14]$. The oscillatory field consists of the collective motion of spatially distributed intracellular biochemical oscillators $[6,27]$. So far, these wave phenomena have been studied by using oscillatory reaction-diffusion equations, but recent experimental results suggest that the overall wave behaviour is strongly affected by the hydrodynamic flow of protoplasm [18]. To take this into account, a reaction-diffusion-advection model was recently proposed for the system and the arising wave behaviour was analysed in $[16,28]$. The flow is protoplasmic streaming, which is derived from a biologically active process and said to be self-consistent [28]. Namely, the flow is not constant but varies with respect to space and time, depending on the state of the system. This is a difficulty and the reason why the wave behaviour is not well understood.

\subsection{Biological background}

The plasmodium is a large aggregate of protoplasm with sheet-like morphology and it shows rhythmic contraction everywhere within the organism. While this cyclic contraction is coupled with oscillations of biochemical components including $\mathrm{Ca}^{2+}$, ATP, $\mathrm{H}^{+}$and NADH, it produces motive force (hydrostatic pressure) leading to protoplasmic streaming [19,26,29-31]. The streaming direction is switched periodically back and forth, depending on the spatial difference of the contraction phase. For these reasons, it is necessary that the plasmodium is regarded as a coupled oscillator system with advection [16,28]. In fact, phase waves of cellular rhythm are observed in the real organism, and play a significant role in regulating the mechanisms controlling chemotaxis, thermotaxis and phototaxis $[13,14,17]$. Although these tactic responses can be explained by the nonlinear dynamics of the phase wave, these phase waves have so far been analysed for a conventional oscillatory reaction-diffusion model, and the effect of the flow has been ignored for the sake of simplicity.

\subsection{Aims and outline}

We begin by motivating our study with some examples of phase waves in Physarum polycephalum. We show the spatio-temporal patterns of rhythmic oscillation that arise in the organism under different external conditions. We then move to take a more theoretical approach: studying, in general, the effects of self-consistent flow on the wave behaviour of oscillatory reaction-diffusion equations. We use numerical techniques to calculate the dispersion relation for propagating waves, and analyse the effects of the flow on travelling plane waves. Later we consider a more general setting: we derive an approximation for the dynamics of phase waves arising in a generic reaction-diffusion-advection model by means of a perturbation method. Finally we suggest possible physiological roles of the self-consistent flow in the Physarum plasmodium. 


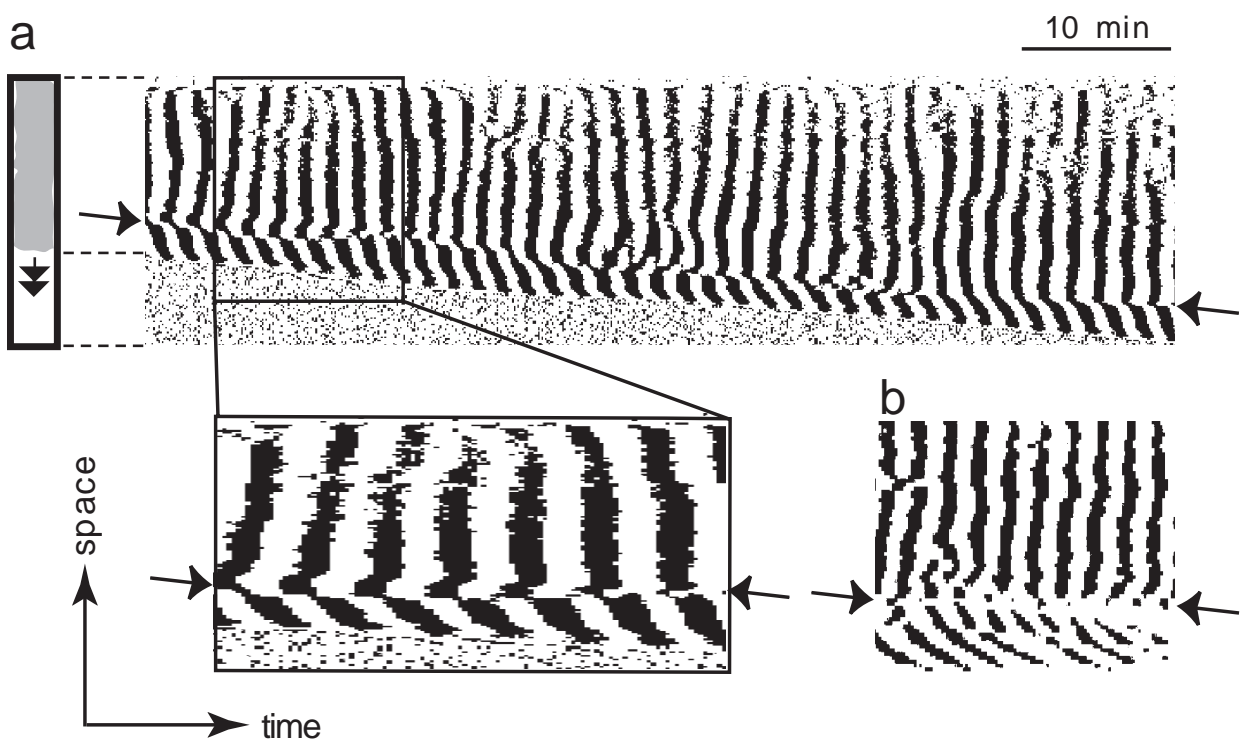

Fig. 1 Acceleration of phase waves in the actual organism. (a) Typical spatio-temporal pattern of rhythmic oscillation, measured by the conventional optical method [13], in a plasmodium extending freely along a lane of agar gel, as shown on the left. In the figure, the organism, indicated by shading, moved downward as indicated by the double arrow. The phase of the oscillation was almost synchronous through the width of the lane. We recorded the time series observed along a middle line of the lane width. The propagation speed of the wave was faster in the rear than in the frontal tip (the boundary between these two regions is indicated). The lower figure, drawn on an enlarged time scale, shows more clearly the difference in propagation speed. (b) Slower wave propagation on a nutrient-containing gel (below the arrows). In all figures, the vertical and horizontal directions indicate space and time, respectively. The oscillation phase of cell thickness was discretised into two states, increase (black) and decrease (white). Scale bar: 10 minutes except for the enlarged figure of (a). Length of organism was 2-4 cm.

\section{Wave acceleration of real organism in relation to variations in protoplasmic streaming}

Figure 1 shows a plasmodium extending freely along a lane of agar gel (see upperleft part of figure): as time progresses the plasmodium extends along the lane, from top to bottom (double arrow). The different parts of Figure 1 show some real patterns of rhythmic contraction in relation to changes in strength of protoplasmic shuttle streaming. In order to demonstrate the oscillations, the phase of cell thickness was discretised into two states: increasing (black) and decreasing (white).

In Figure 1(a) we see that in the frontal tip of the freely extending organism (below an imaginary line connecting the two horizontal arrows), wave propagation is slower than in the rear part of the organism (above the imaginary line). This slow propagation arises as a result of the changes in the protoplasmic flow rate: weaker in the frontal tip of the organism. The phenomenon can be observed more clearly in chemotaxis of the organism, as shown in Figure 1(b). On the nutrient site (below the imaginary line connecting the arrows), the wave speed is much slower: it is known that protoplasmic flow is weaker at nutrient-rich sites than at 
(a) Concentration profiles

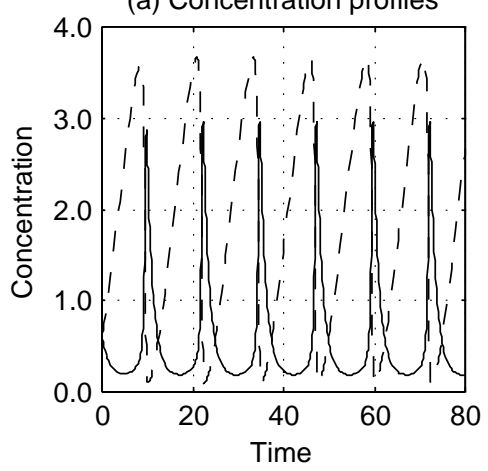

(b) Phase diagram

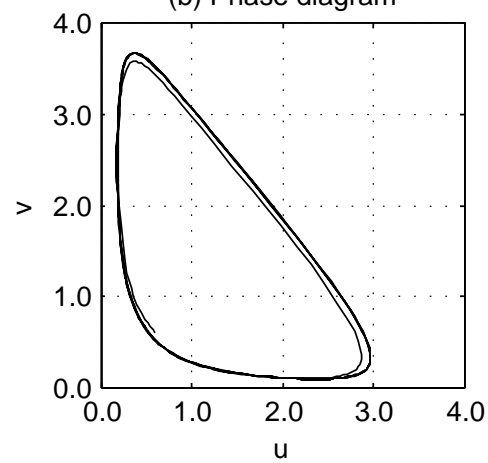

Fig. 2 Numerical solution of the spatially homogeneous system $(D, w=0$ in equation (1)) with Schnakenberg kinetics. (a) shows the concentration profiles of $u$ (solid line) and $v$ (dashed line) over time. (b) is a phase diagram and clearly shows that the system quickly tends to a limit cycle. Parameters are as follows: $a=0.1, b=0.5, u(0)=0.6, v(0)=0.6$ and both plots are shown for $t \in[0,80]$.

other sites (above the imaginary line). From these results, we conclude that wave propagation is accelerated by increased protoplasmic flow.

These findings motivate our study: we investigate the effects of flow on wave propagation rates in oscillatory reaction-diffusion systems, in order to determine whether protoplasmic streaming rates can affect the phase waves observed in the plasmodium.

\section{Numerical calculation of the dispersion relation}

A recently proposed model for contraction dynamics of the Physarum plasmodium is a system of reaction-diffusion equations with flow terms $[7,16,28]$. To numerically calculate a dispersion relation, we specify a conventional two-variable model with self-consistent flow [28]:

$$
\begin{aligned}
\frac{\partial u}{\partial t}+w \cdot \nabla u & =f(u, v)+D \nabla^{2} u \\
\frac{\partial v}{\partial t} & =g(u, v)
\end{aligned}
$$

where $u$ and $v$ are chemical concentrations. Here, we assume that one metabolic chemical described by $u$ flows with the endoplasmic streaming while the other chemical described by $v$ is bound to ectoplasm. The quantity $D$ is the diffusion constant of the chemical $u$. The velocity of the self-consistent flow, $w$, is determined by the concentration of the metabolic chemical as

$$
w=q \nabla u,
$$

where $q$ is a (constant) parameter expressing the intensity of the flow. 
Model equations 1 and 2 take the form of a generalized reaction-diffusionadvection model with self-consistent flow proposed in [28] but they can be obtained by some simplification from a previously proposed model with three components [16]. In the previous model, the variables $u$ and $v$ are chemicals which display oscillatory behavior in ectoplasm. While $v$ is bound in the ectoplasm, $u$ is a free component which can interact with contractile proteins and exchanges with the same chemical in flowing endoplasm (say $z$ ). The dynamics of $z$ has an advection term dependent on $u$. Although this exchange of $u$ and $z$ is not so fast, we assume, for the sake of simplicity, that $u$ is always similar to $z$ and has an advection term in addition to its intrinsic diffusion term. Our justification for this comes from the fact that we make the advection coefficient, $q$, small enough. From this, equation 1 is obtained.

The reaction kinetics are chosen to exhibit a limit cycle oscillation. Here the functions $f$ and $g$ are taken to be the Schnakenberg (tri-molecular) reaction kinetics $[15,24]$ :

$$
f(u, v)=a-u+u^{2} v, \quad g(u, v)=b-u^{2} v,
$$

where $a$ and $b$ are positive constants. Under spatially homogeneous conditions $(D=0$ and $w=0)$, the system has a stable limit cycle for $b-a>(a+b)^{3}[15]$. We chose this reaction system because the shape of the limit cycle in the phase space is similar to that of the calcium oscillation in the plasmodium, which is a strong candidate for the primary chemical clock underlying the rhythmic activity. Figure 2 shows plots of the numerical solution of the spatially homogeneous system and clearly demonstrates the limit cycle kinetics.

Plane wave solutions of the system given by equations (1)-(3) are of the form

$$
u(x, t)=U(\omega t-k x) \quad \text { and } \quad v(x, t)=V(\omega t-k x),
$$

where $\omega$ is the wave frequency (so that the period $T=2 \pi / \omega$ ) and $k$ is the wave number (so that the wave length $\lambda=2 \pi / k$ ). The propagation velocity can be calculated as $v=\omega / k$.

To obtain the dispersion relation for travelling waves, we carried out numerical calculations for equations (1)-(3) with the following procedure. We initiated a pulse travelling on a ring (a one-dimensional region with the periodic boundary conditions), and solved the system until the solution became periodic in time. After measuring the rotating period of the travelling pulse on the ring, we repeated the calculation for rings of different lengths. Thus we obtained the dispersion relation for periodic wave trains with stable propagation.

We used the explicit Euler method for the reaction terms, an upwind differencing method for the advection terms, and an implicit method for the diffusion terms. A more detailed outline of the numerical calculations, including the initial conditions and numerical discretisation employed, can be found in Appendix A. In our calculations, the parameter values were taken to be $a=0.1, b=0.5$ and $D=1.0$. To see if these results are typical of the behaviour of the system, we undertook an analytical investigation: it is time consuming to carry out a detailed numerical investigation over a wide parameter space. The analysis outlined in Section 4 will allow us to approximate the dispersion relation by numerical calculation of just two integrals. In this way we can quickly investigate whether the behaviour observed with the parameter set of Figure 3 is representative of that observed for more general parameter choices. 
(a) Concentration profile for $u$

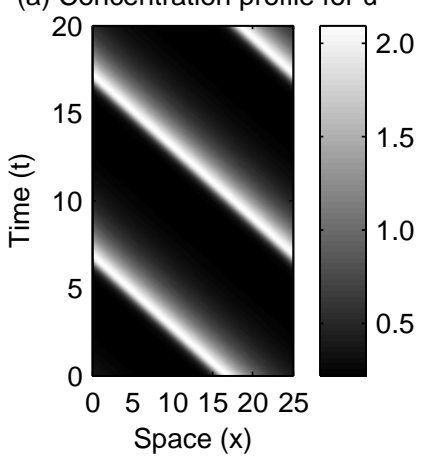

(b) Concentration profile for $v$

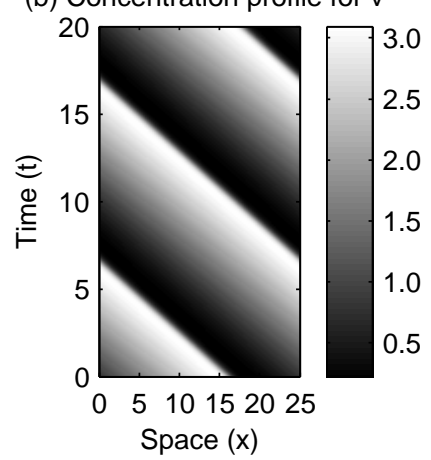

Fig. 3 Numerical solution of the given by equations (1)-(3). The numerical method is outlined in Appendix A. (a) shows the concentration profile for $u$ over $(x, t)$-space while (b) shows the concentration profile for $v$. Parameters are as follows: $a=0.1, b=0.5, q=1.0$ and $D=1.0$.

Figure 3 shows a plot of the numerical solution of equations (1)-(3) and clearly demonstrates the periodic behaviour of $u$ and $v$. Plane waves with a wave length of $\lambda=25(k \approx 0.25)$ occur with a period of approximately $T=10(\omega \approx 0.63)$.

Dispersion curves for the reaction-diffusion-advection system, given by equations (1)-(3), are shown in Figure 4 (wave number, $k$, against frequency, $\omega$ ) and Figure 5 (period, $T=2 \pi / \omega$, against velocity, $v=\omega / k$ ) for various values of the advection constant, $q$. It can clearly be seen from the figures that variations in the self-consistent flow have a pronounced effect on plane wave propagation, and that propagation behaviour depends on both the wave length and on the sign of $q$.

Following [1,2,23], we classify our waves into phase waves (long wavelength, low wave number) and trigger waves (short wavelength, high wave number) by two branches of the curve separated by the inflection point. These classifications have been marked on both Figure 4 and Figure 5 .

In both cases ( $q$ positive and negative), as $q$ is increased (while the wave number, $k$, is kept constant) the oscillation frequency of phase waves, $\omega$, remains essentially constant (note that the plots of Figure 4 are on different scales). However, the change in frequency is marked for trigger waves. As $q$ is increased, the oscillation frequency of trigger waves decreases: the change in frequency is more significant for $q<0$. We note that this implies that as the modulus of the flow is increased, opposite effects occur according to whether the flow is in a positive or negative direction. For example, for trigger waves and $q>0, \omega$ decreases as $|q|$ increases, while for $q<0, \omega$ increases as $|q|$ increases.

In contrast we see that as $q$ is increased (while the oscillation frequency, $\omega$, is kept constant) the wave number, $k$, of trigger waves increases. Again we note that this results in opposing effects when considering the modulus (i.e. the 'strength') of the flow. 

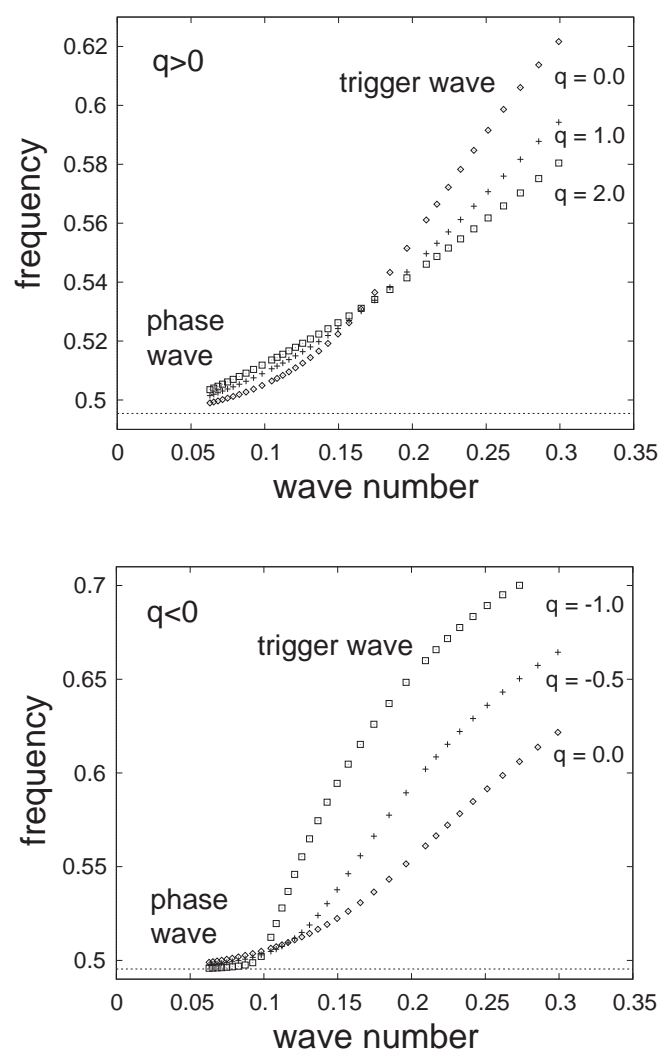

Fig. 4 Dispersion curves obtained by numerical calculations for positive (upper panel) and negative (lower panel) values of $q$. The oscillation frequency, $\omega$, monotonically increases with the wave number, $k$, and has bulk frequency $\omega_{0}=0.495$ in the limit $k \rightarrow 0$.

\subsection{Application to Physarum plasmodium}

In the previous section, the parameter $q$, expressing the intensity of flow, was shown to be crucial to the rate of wave propagation. This was the coefficient through which the chemical oscillator was related to the motive force of protoplasmic flow. Therefore, $q$ is associated with the power which is produced by the mechano-chemical apparatus of actomyosin in the organism. A recent simulation for the rhythmic amoeboid movement in Physarum plasmodium claimed that this type of parameter, which in that report was called the 'stiffness of ectoplasmic gel', played a key role in determining the speed and stabilisation of wave propagation [7,25]. This also indicates that the coefficient of flow or stiffness is a key parameter for regulation of phase wave propagation.

In the actual case of the Physarum plasmodium $q$ is positive, provided that the contraction force is maximum when chemical concentration is minimum. Such behaviour is observed for $\mathrm{Ca}^{2+}$, which is the most probable candidate for a primary chemical clock in the plasmodium [16]. 

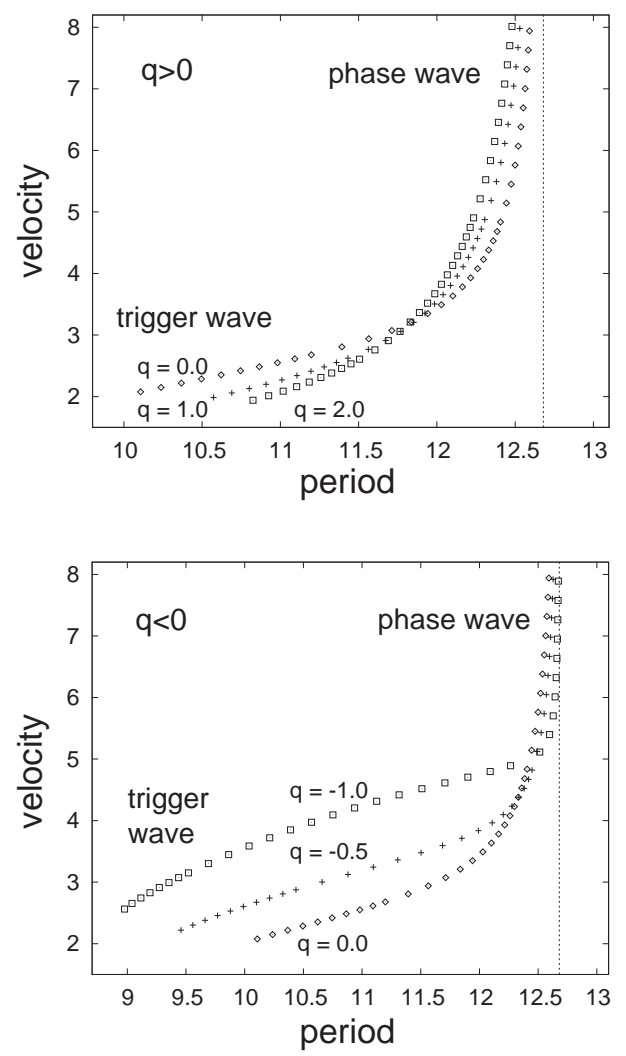

Fig. 5 Relation between period, $T$, and velocity, $v$. The propagation velocity, $v$, was determined as the phase velocity $\omega / k$ for the positive (upper panel) and negative (lower panel) $q$. The velocity becomes large and goes to infinity at the bulk period $\tau_{0}=12.7$. For negative values of $q$, the curves show large convex regions in period corresponding to trigger waves.

Hereafter, we will focus only on the phase wave which is characterised by a low wave number, $k$, since only low wave numbers were observed in the actual organism. The results of this section show that self-consistent flow has a number of profound effects on the phase waves, such as acceleration of the wave. This can be thought of as equivalent to elongation of the wave length, since the frequency of the phase waves remains almost unchanged. Clearly this effect has advantages for intracellular signal communication because physiological information is encoded in the phase of oscillation $[13,14]$. By making use of the acceleration, the plasmodium can maintain a system as large as up to the order of a metre, for example, in spite of it being a unicellular organism with no nervous system. 


\section{Derivation of phase dynamics and estimation of dispersion relation for a generic model}

In this section, we consider a general type of reaction-diffusion system with flow terms [28]:

$$
\frac{\partial \mathbf{u}}{\partial t}+M \nabla \mathbf{u} \cdot \nabla \mathbf{u}=\mathbf{f}(\mathbf{u})+D \nabla^{2} \mathbf{u}
$$

where $\mathbf{u}$ is an $N$-component vector of chemical concentrations varying over space, $x$, and time, $t$. The reaction kinetics, denoted by $\mathbf{f}$, are of limit cycle type and the quantity $D$ is a positive diagonal matrix of diffusion constants. The velocity of chemical flow resulting from protoplasmic streaming is determined by the concentration of the chemicals as $M \nabla \mathbf{u}$, where the tensor $M$ represents advection coefficients. To consider the effect of flow on the dispersion relation, we derive the phase dynamics from equation (5), and estimate a dispersion relation for the phase wave.

By means of limit cycle perturbations, the dynamics of phase waves in standard reaction-diffusion systems for oscillatory media are described by Burgers equation [10,11,20-22]. We adopt a similar method for oscillatory reaction-diffusion equations with this type of flow term.

We assume that the limit cycle is described by a solution of equation (5) under spatially homogeneous oscillation with frequency $\omega_{0}$ :

$$
\mathbf{u}=\mathbf{u}_{0}(\tau), \quad \tau=\omega_{0} t,
$$

where $\mathbf{u}_{0}$ satisfies $\omega_{0} \mathbf{u}_{0}^{\prime}=\mathbf{f}\left(\mathbf{u}_{0}\right)$ and $\mathbf{u}_{0}(\tau+2 \pi)=\mathbf{u}_{0}(\tau)$. Since the system (5) is invariant under time translation, it has a solution $\mathbf{u}=\mathbf{u}_{0}(\tau+\psi)$ where $\psi$ is an arbitrary constant.

We introduce multiple scales

$$
X=\sqrt{\varepsilon} x, \quad \tau=\omega_{0} t, \quad T=\varepsilon t,
$$

and the asymptotic expansion,

$$
\mathbf{u}=\mathbf{u}_{0}(\tau+\psi)+\varepsilon \mathbf{u}_{1}(\tau+\psi)+\ldots,
$$

where $\varepsilon$ is a small parameter and $\psi=\psi(X, T)$. Substitution of equations (7) and (8) into equation (5) yields a hierarchy of linear equations for each order in $\varepsilon$ :

$$
\begin{aligned}
\omega_{0} \frac{\partial \mathbf{u}_{0}}{\partial \tau} & =\mathbf{f}\left(\mathbf{u}_{0}\right), \\
\mathscr{L} \mathbf{u}_{j} & =\mathbf{b}_{j},
\end{aligned}
$$

where

$$
\mathscr{L}=\omega_{0} \frac{\partial}{\partial \tau}-\frac{\partial \mathbf{f}}{\partial \mathbf{u}}\left(\mathbf{u}_{0}\right)
$$

Here $\mathbf{b}_{j}$ denotes the inhomogeneous term of the $j$ th order equation for $j=1,2, \ldots$. For the first order equation, the inhomogeneous term is

$$
\begin{aligned}
\mathbf{b}_{1} & =-\frac{\partial \mathbf{u}_{0}}{\partial T}-M \nabla_{X} \mathbf{u}_{0} \cdot \nabla_{X} \mathbf{u}_{0}+D \nabla_{X}^{2} \mathbf{u}_{0} \\
& =-\mathbf{u}_{0}^{\prime} \frac{\partial \psi}{\partial T}-M \mathbf{u}_{0}^{\prime} \mathbf{u}_{0}^{\prime}\left|\nabla_{X} \psi\right|^{2}+D \mathbf{u}_{0}^{\prime \prime}\left|\nabla_{X} \psi\right|^{2}+D \mathbf{u}_{0}^{\prime} \nabla_{X}^{2} \psi
\end{aligned}
$$


where $\nabla_{X}$ is the nabla operator with respect to the scaled coordinate $X$. Thus the solvability condition for $\mathbf{u}_{1}$ gives the dynamics of the phase waves:

$$
\frac{\partial \psi}{\partial T}=c_{1} \nabla_{X}^{2} \psi+c_{2}\left|\nabla_{X} \psi\right|^{2}
$$

The coefficients $c_{1}$ and $c_{2}$ are obtained from the relations,

$$
c_{j}=\frac{\left\langle\mathbf{v}^{\dagger}, \mathbf{v}_{j}\right\rangle}{\left\langle\mathbf{v}^{\dagger}, \mathbf{u}_{0}^{\prime}\right\rangle},
$$

where

$$
\mathbf{v}_{1}=D \mathbf{u}_{0}^{\prime}, \quad \text { and } \quad \mathbf{v}_{2}=D \mathbf{u}_{0}^{\prime \prime}-M \mathbf{u}_{0}^{\prime} \mathbf{u}_{0}^{\prime}
$$

Here

$$
\left\langle\mathbf{v}^{\dagger}, \mathbf{v}\right\rangle=\int_{0}^{2 \pi}\left(\mathbf{v}^{\dagger}, \mathbf{v}\right) d \tau
$$

and $\mathbf{v}^{\dagger}$ is the nontrivial periodic solution to the adjoint differential equation $\mathscr{L}^{\dagger} \mathbf{v}^{\dagger}=$ 0. Equation (14) describes slow modulation of the homogeneous oscillation with frequency $\omega_{0}$ by the phase $\psi$. We note that the coefficient of the nonlinear term, $c_{2}$, shows 'competition' between diffusion and flow.

In terms of the quantity $\phi=\omega_{0} t+\psi$, equation (14) becomes

$$
\frac{\partial \phi}{\partial t}=\omega_{0}+c_{1} \nabla^{2} \phi+c_{2}|\nabla \phi|^{2}
$$

The dispersion relation is thus estimated from the phase equation (18) through the wave characteristics $\omega=\partial \phi / \partial t$ and $\mathbf{k}=\nabla \phi$ as $[1,2]$

$$
\omega=\omega_{0}+c_{2} k^{2}+\ldots
$$

where $k=|\mathbf{k}|$.

Since the scaling of coordinates in the perturbation expansions (7) means a slow spatial modulation, $k=O(\sqrt{\varepsilon})$, equation (19) is the Taylor expansion for the dispersion curve, $\omega=\omega(k)$, in the vicinity of $k=0$. Thus, the coefficient of the nonlinear term in equation (18) is $c_{2}=\omega^{\prime \prime}(0) / 2$. Here equation (19) has no linear term in $k$ because of reflectional symmetry in the space of equation (5). As shown above in equation (16), $c_{2}$ depends on advection constants as well as diffusion constants.

The dispersion relation, equation (19), shows that the wave number, $k$, can decrease at a fixed value of the frequency $\omega$, as $c_{2}$ varies, depending on the flow term. But $c_{2}$ also depends on the diffusion term and the concrete form of the function was given by equation (16).

We note that the dispersion relation, (19), is only applicable to periodic waves with constant speed. It does not apply to waves with non-uniform phase gradients [23]. In such cases, we need to use the phase equation, (18), or analyse equation (5) directly. 
4.1 Application to the Physarum plasmodium model

We now apply the results of this section to determine an approximate dispersion relation for the model of Section 3. From equations (1)-(3) we see that the operator $\mathscr{L}$ and its adjoint, $\mathscr{L}^{\dagger}$ are given by:

$$
\mathscr{L}=\left(\begin{array}{cc}
\omega_{0} \frac{\partial}{\partial \tau}-\frac{\partial f}{\partial u}\left(u_{0}, v_{0}\right) & -\frac{\partial f}{\partial v}\left(u_{0}, v_{0}\right) \\
-\frac{\partial g}{\partial u}\left(u_{0}, v_{0}\right) & \omega_{0} \frac{\partial}{\partial \tau}-\frac{\partial g}{\partial v}\left(u_{0}, v_{0}\right)
\end{array}\right),
$$

and

$$
\mathscr{L}^{\dagger}=\left(\begin{array}{cc}
-\omega_{0} \frac{\partial}{\partial \tau}-\frac{\partial f}{\partial u}\left(u_{0}, v_{0}\right) & -\frac{\partial g}{\partial u}\left(u_{0}, v_{0}\right) \\
-\frac{\partial f}{\partial v}\left(u_{0}, v_{0}\right) & -\omega_{0} \frac{\partial}{\partial \tau}-\frac{\partial g}{\partial v}\left(u_{0}, v_{0}\right)
\end{array}\right) .
$$

This results in a solvability condition of

$$
\begin{aligned}
0= & \int_{0}^{2 \pi} u_{0}^{\dagger}\left[-u_{0}^{\prime} \frac{\partial \psi}{\partial T}-q\left(u_{0}^{\prime}\right)^{2}\left|\nabla_{X} \psi\right|^{2}+D u_{0}^{\prime \prime}\left|\nabla_{X} \psi\right|^{2}+D u_{0}^{\prime}\left|\nabla_{X}^{2} \psi\right|\right] d \tau \\
& +\int_{0}^{2 \pi} v_{0}^{\dagger}\left[-v_{0}^{\prime} \frac{\partial \psi}{\partial T}\right] d \tau,
\end{aligned}
$$

where $u_{0}, v_{0}, u_{0}^{\dagger}$ and $v_{0}^{\dagger}$ satisfy the equations

$$
\omega_{0}\left(\begin{array}{c}
\frac{\partial u_{0}}{\partial \tau} \\
\frac{\partial v_{0}}{\partial \tau}
\end{array}\right)=\left(\begin{array}{c}
f\left(u_{0}, v_{0}\right) \\
g\left(u_{0}, v_{0}\right)
\end{array}\right)
$$

and

$$
-\omega_{0}\left(\begin{array}{c}
\frac{\partial u_{0}^{\dagger}}{\partial \tau} \\
\frac{\partial v_{0}^{\dagger}}{\partial \tau}
\end{array}\right)=\left(\begin{array}{l}
\frac{\partial f}{\partial u}\left(u_{0}, v_{0}\right) \frac{\partial g}{\partial u}\left(u_{0}, v_{0}\right) \\
\frac{\partial f}{\partial v}\left(u_{0}, v_{0}\right) \frac{\partial g}{\partial v}\left(u_{0}, v_{0}\right)
\end{array}\right)\left(\begin{array}{c}
u_{0}^{\dagger} \\
v_{0}^{\dagger}
\end{array}\right) .
$$

From this we have a dispersion relation of the form

$$
\omega=\omega_{0}+c_{2} k^{2}+\ldots
$$

for small $k$, where

$$
c_{2}=\int_{0}^{2 \pi} u_{0}^{\dagger}\left[D u_{0}^{\prime \prime}-q\left(u_{0}^{\prime}\right)^{2}\right] d \tau / \int_{0}^{2 \pi}\left[u_{0}^{\prime} u_{0}^{\dagger}+v_{0}^{\prime} v_{0}^{\dagger}\right] d \tau .
$$

Since equations (23) and (24) cannot be solved analytically, we employ numerical techniques to gain an estimate of the coefficient $c_{2}$ for varying values of the flow parameter, $q$. We solve the system of differential equations in Matlab and then evaluate the integrals by employing the trapezoidal rule. The dispersion relation for differing values of $q$ is plotted in Figure 6.

Application of the method of Section 4 to the Physarum plasmodium model neatly demonstrates the competition between rates of diffusion, $D$, and advection, $q$ in determining the dispersion relation (see equation (26)). However, without the aid of numerical tools, it is difficult to make remarks (either qualitative or quantitative) on the behaviour of $c_{2}$ as $D$ and $q$ are varied. This is due to the highly nonlinear nature of the problem: the effects of varying $q$ and $D$ will depend specifically on the problem at hand. 


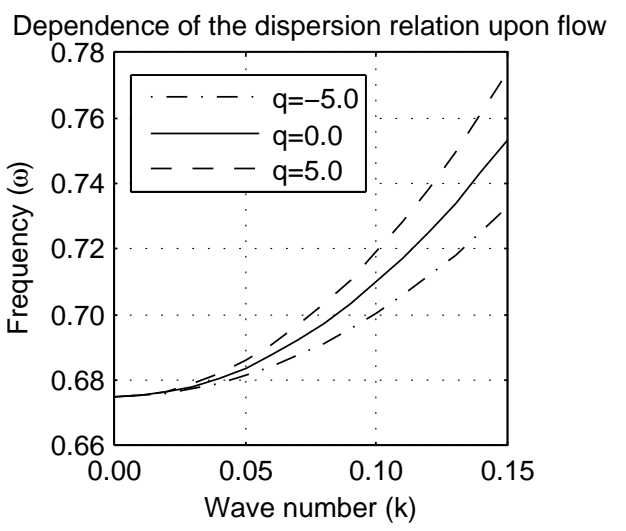

Fig. 6 Dependence of the dispersion relation upon the flow. Equations (23) and (24) were solved numerically and then the parameter $c_{2}$ was calculated for varying values of the flow parameter, $q$. As $q$ is increased the frequency, $\omega$, increases for fixed values of the wave number, $k$. We note that this is in agreement with numerical simulations of the system shown in Figures 4 and 5. Parameters were as follows: $a=0.18, b=0.5$ and $D=1.0$.

\section{Conclusion}

We began by motivating our theoretical studies with observation of wave propagation rates in the actual organism, Physarum plasmodium. From consideration of the spatio-temporal patterns of rhythmic oscillation we inferred that decreased protoplasmic flow results in a decrease in wave propagation speed.

We then studied the effects of self-consistent flow on wave behaviour of the oscillatory reaction-diffusion model proposed for the Physarum plasmodium. We used numerical techniques to solve the system of equations (1)-(3), and through this obtained a dispersion relation for varying values of the flow rate, $q$, and wave number, $k$. The addition of self-consistent flow had a number of profound effects on the phase waves, such as acceleration of the wave. We noted that this is equivalent to elongation of the wave length since the frequency of the phase waves remained almost unchanged.

These theoretical results are in agreement with the inferences of Section 2 regarding the actual organism: the protoplasmic streaming was able to accelerate the phase wave speed. As discussed in the text, it is clear this effect could provide advantages for intracellular signal communication due to the fact that physiological information is encoded in the phase of oscillation.

To consider the effect of flow on the dispersion relation in a more general setting, we derived phase dynamics for a generic reaction-diffusion system, given by equation (5) and used asymptotic techniques to derive an estimate of the dispersion relation for phase waves. The dispersion relation, equation (19), showed that the wave number decreased at a fixed value of frequency as $c_{2}$ varied depending on the flow term. The flow effect on wave acceleration could be understood by this generic relation. We concluded that the flow term was able to accelerate the phase wave speed (similar to elongation of wave length). 
Acknowledgements TN is supported by the Grant-in-Aid for Scientific Research, No.15300098, the Japan Society for the Promotion of Science. REB would like to thank Lloyds Tercentenary Foundation for a Lloyds Tercentenary Foundation Fellowship, Research Councils UK for an RCUK Academic Fellowship in Mathematical Biology and St Hugh's College, Oxford for a Junior Research Fellowship.

\section{A Numerical methods}

The system solved numerically in Section 3 was of the form:

$$
\begin{aligned}
\frac{\partial u}{\partial t}+q \nabla u . \nabla u & =f(u, v)+D \nabla^{2} u, \\
\frac{\partial v}{\partial t} & =g(u, v),
\end{aligned}
$$

where $f$ and $g$ are as in equation (3). The system was solved on the domain $x \in[0, \lambda]$, with periodic boundary conditions: $u(0, t)=u(\lambda, t)$, and similarly for $v$. The initial conditions were taken in the form of a 'pulse':

$$
\begin{aligned}
& u(x, t)=\frac{1}{2}+\sin \left(\frac{2 \pi x}{\lambda}\right), \\
& v(x, t)=\frac{1}{2}+\frac{9}{5} \cos \left(\frac{2 \pi x}{\lambda}\right) .
\end{aligned}
$$

The system was discretised using the following methods: explicit Euler method for the reaction terms; upwind method for the advection term; leapfrog method for the flow, w, and implicit method for the diffusion terms.

Letting $U_{j}^{n} \approx u\left(x_{j}, t_{n}\right)$ and $V_{j}^{n} \approx v\left(x_{j}, t_{n}\right)$, where $x_{j+1}-x_{j}=\Delta x$ and $t^{n+1}-t^{n}=\Delta t$, results in the following discretised system:

$$
\begin{aligned}
\frac{U_{j}^{n+1}-U_{j}^{n}}{\Delta t}+q\left[\frac{U_{j+1}^{n}-U_{j-1}^{n}}{2(\Delta x)}\right]\left[\frac{U_{j+1}^{n}-U_{j}^{n}}{\Delta x}\right] & =f\left(U_{j}^{n}, V_{j}^{n}\right)+D\left[\frac{U_{j+1}^{n+1}-2 U_{j}^{n+1}+U_{j-1}^{n+1}}{(\Delta x)^{2}}\right] \\
\frac{V_{j}^{n+1}-V_{j}^{n}}{\Delta t} & =g\left(U_{j}^{n}, V_{j}^{n}\right) .
\end{aligned}
$$

For each value of the flow parameter, $q$, accuracy was ensured by suitably refining the spatial and temporal mesh parameters.

\section{References}

1. Aliev, R. R.: Oscillation phase dynamics in the Belousov-Zhabotinsky reaction - implementation to image-processing. J. Phys. Chem. 98, 3999-4002 (1994).

2. Aliev, R. R., and Biktashev, V. N.: Dynamics of the oscillation phase distribution in the BZ reaction. J. Phys. Chem. 98, 9676-9681 (1994).

3. Greenberg, J. M.: Spiral waves for $\lambda$ - $\omega$ systems, II. Adv. Appl. Math. 2, 450-455 (1981).

4. Hagan, P. S.: Spiral waves in reaction-diffusion equations. SIAM J. Appl. Math. 42, 762-786 (1982).

5. Howard, L. N., and Kopell, N.: Slowly varying waves and shock structures in reactiondiffusion equations. Stud. Appl. Math. 56, 95-145 (1977).

6. Kamiya, N.: Protoplasmic streaming. Protoplasmatologia 8, 1-199 (1959).

7. Kobayashi,R., Tero, A., and Nakagaki, T.,: Mathematical model for rhythmic amoeboid movement in the true slime mold. J. Math. Biol., 53, 273-286 (2006).

8. Koga, S.: Rotating spiral waves in reaction-diffusion systems - phase singularities of multiarmed waves. Prog. Theor. Phys. 67, 164-178 (1982). 
9. Kopell, N., and Howard, L. N.: Plane-wave solutions to reaction-diffusion equations. Stud. Appl. Math. 52, 291-328 (1973).

10. Kuramoto, Y., and Tsuzuki, T.: Formation of dissipative structures in reaction-diffusion systems - reductive perturbation approach. Prog. Theor. Phys. 54, 687-699 (1975).

11. Kuramoto, Y. and Yamada, T.: Pattern formation in oscillatory chemical-reactions. Prog. Theor. Phys. 56, 724-740 (1976).

12. Kuramoto, Y., and Koga, S.: Turbulized rotating chemical waves. Prog. Theor. Phys. 66, 1081-1085 (1981).

13. Matsumoto, K., Ueda, T., and Kobatake, Y.: Reversal of thermotaxis with oscillatory stimulation in the plasmodium of Physarum polycephalum. J. Theor. Biol. 131, 175-182 (1988).

14. Miyake, Y., Tabata, S., Murakami, H., Yano, M., and Shimizu, H.: Environment-dependent self-organization of positional information field in chemotaxis of Physarum polycephalum. J. Theor. Biol. 178, 341-353 (1996).

15. Murray, J. D.: Mathematical Biology. Springer (1989).

16. Nakagaki, T., Yamada, H., and Masami, I.: Reaction-diffusion-advection model for pattern formation of rhythmic contraction in a giant amoeboid cell of the Physarum. plasmodium. J. Theor. Biol. 197, 497-506 (1999).

17. Nakagaki,T., Yamada, H., and Ueda, T.: Modulation of cellular rhythm and photoavoidance by oscillatory irradiation in the Physarum plasmodium. Biophys Chem. 82, 23-28 (1999).

18. Nakagaki, T., Yamada, H., and Ueda, T.: Interaction between cell shape and contraction pattern in the Physarum plasmodium. Biophys. Chem. 84, 195-204 (2000).

19. Nakamura, S., Yoshimoto, Y., and Kamiya, N.: Oscillation in surface $\mathrm{pH}$ of the Physarum plasmodium. Proc. Japan Acad. 58, 270-273 (1982).

20. Ortoleva, P., and Ross, J.: Phase waves in oscillatory chemical reactions. J. Chem. Phys. 58 5673-5680 (1973).

21. Ortoleva, P., and Ross, J.: Variety of wave phenomena in chemical-reactions. J. Chem. Phys. 60, 5090-5107 (1974).

22. Ortoleva, P.: Local phase and renormalized frequency in inhomogeneous chemioscillations. J. Chem. Phys. 64, 1395-1406 (1976).

23. Polezhaev, A. A.: Phase waves in oscillatory media. Physica D 84, 253-259 (1995)

24. Schnackenberg, J.: Simple chemical-reaction systems with limit-cycle behavior. J. Theor. Biol. 81, 389-400 (1979).

25. Tero, A., Kobayashi, R., and Nakagaki, T., Physica D, in press (2005).

26. Ueda, T.: Intracellular oscillations and pattern formation in the cell behavior of Physarum. In: Rensing, L., (ed.) Oscillations and morphogenesis. 167-181, Marcel-Dekker, New York (1993).

27. Ueda, T., Matsumoto, K., Akitaya, T., and Kobatake, Y.: Spatial and temporal organization of intracellular adenosine nucleotide and cyclic nucleotides in relation to rhythmic motility in Physarum polycephalum. Exp. Cell Res. 162, 486-494 (1986).

28. Yamada, H., Nakagaki, T., and Ito, M.: Pattern formation of a reaction-diffusion system with self-consistent flow in the amoeboid organism Physarum plasmodium. Phys. Rev. E 59, 1009-1014 (1999).

29. Yoshimoto, Y., Matsumura, F., and Kamiya, N.: Simultaneous oscillations of $\mathrm{Ca}^{2+}$ efflux and tension generation in the permealized plasmodial strand of Physarum. Cell Motility $\mathbf{1}$, 433-443 (1981).

30. Yoshimoto, Y., Sakai, T., and Kamiya, N.: ATP oscillation in Physarum plasmodium. Protoplasm. 109, 159-168 (1981).

31. Yoshimoto, Y., Sakai, T., and Kamiya, N.: ATP- and calcium-controlled contraction in a saponin model of Physarum polycephalum. Cell Struct. Funct. 9, 135-141 (1984). 